\title{
Tumor Microenvironment-responsive Nanodrug for Clear-cell Renal Cell Carcinoma Therapy via Triggering Waterfall-like Cascade Ferroptosis
}

\section{Wenjun Ni}

Zhuhai People's Hospital, Zhuhai Hospital Affiliated with Jinan University, Jinan University

\section{Yongxiang Li}

Weifang People's Hospital

Lingxia Liang

Medical College of Guangxi University, Guangxi University

Cuixia Lu

Medical College of Guangxi University, Guangxi University

\section{Meixiao Zhan}

Zhuhai People's Hospital, Zhuhai Hospital Affiliated with Jinan University, Jinan University

\section{Ligong Lu}

Zhuhai People's Hospital, Zhuhai Hospital Affiliated with Jinan University, Jinan University

\section{Qun Xie}

Zhuhai People's Hospital, Zhuhai Hospital Affiliated with Jinan University, Jinan University

Liewei Wen ( $\nabla$ wenliewei@scnu.edu.cn )

Zhuhai People's Hospital, Zhuhai Hospital affiliated with Jinan University, Jinan University https://orcid.org/0000-0002-4657-6457

\section{Research}

Keywords: Renal cell carcinoma, ferroptosis, metal organic frameworks, lipid peroxides

Posted Date: September 1st, 2021

DOI: https://doi.org/10.21203/rs.3.rs-842110/v1

License: (c) (1) This work is licensed under a Creative Commons Attribution 4.0 International License. Read Full License 


\section{Abstract}

\section{Background}

The most common type of kidney tumor, clear-cell renal cell carcinoma (ccRCC) with relatively insidious development and easily metastatic characteristics is generally insensitive to cytotoxic chemotherapy. Advanced ccRCC lacks effective treatment and has a poor prognosis. Fortunately, ccRCC with the hallmark of abundant polyunsaturated fatty acids (PUFAs) content is regarded as intrinsically vulnerable to ferroptosis-based therapeutic strategies. Nonetheless, the classic ferroptosis agonist (RSL3) with low specificity for tumors, short half-life in the blood, poor water solubility and deficient accumulation at the tumor site prevents its reliable application in vivo.

Results

In this study, iron-based metal-organic framework nanoparticles (MIL-101(Fe) NPs) delivered RSL3 to ccRCC tumors, and then released the iron ions and RSL3 accompanied by the degradation of MIL-101(Fe) NPs in the acidic tumor microenvironment. The MIL-101(Fe)@RSL3 as a pH-responsive nanodrug causes cellular iron overload and promotes the hydroxyl radical $(\cdot \mathrm{OH})$ generation by Fenton reaction to attack PUFAs, leading to the aberrant accumulation of lipid peroxides $(\mathrm{L}-\mathrm{OOH})$. Additionally, RSL3 directly inhibits glutathione peroxidase 4 (GPX4) to detoxify $\mathrm{L}-\mathrm{OOH}$, and ferrous ions further catalyze the irreversible conversion of highly reactive lipid alkoxyl radicals (L-O•) from L-OOH to triggering waterfalllike cascade ferroptosis. In contrast to the limited antitumor efficiency of free RSL3, MIL-101(Fe)@RSL3 with high encapsulation efficiency (88.7\%) shows a significant ccRCC-specific antitumor effect and negligible side effects.

Conclusion

MIL-101(Fe)@RSL3 could aggravate ferroptosis and be expected to be a promising nanodrug for ccRCC systemic therapy due to the targeted delivery and responsive release of RSL3 and iron ions.

\section{Introduction}

Renal cell carcinoma (RCC), one of the most common genitourinary malignant tumors, causes 170,000 deaths each year worldwide. Clear cell renal cell carcinoma (ccRCC) accounts for more than $75 \%$ of RCC cases, which is characterized by high invasiveness and is inclined to metastasize.[1, 2] However, the treatment of ccRCC remains a challenge in clinical setting, owing to the facts that it is easy to develop metachronous distant metastases after surgical resection, and insensitive to chemotherapy or radiotherapy. $[3,4]$ Therefore, it is still an urgent clinical need for advanced ccRCC to develop the new drugs and treatment strategies.

As far as we know, metabolic reprogramming is an important feature of ccRCC, which is usually associated with mutations in VHL tumor suppressor genes, and about $90 \%$ of ccRCC have VHL 
mutations.[5] Studies showed that mutations in the VHL gene can lead to the stable expression of hypoxia-inducible factors HIF-1 $a$ and HIF-2a.[6] In addition to the aberrant polyunsaturated fatty acids (PUFAs) elevation mediated by HIF-2a, the stable expression of HIF-1 $a$ and HIF-2 $a$ also reduce the catabolism of fatty acids through inhibiting $\beta$ oxidation,[7] endowing the significantly higher level of PUFAs in ccRCC than that of normal kidney tissue.[8] It is worth noting that PUFAs containing diallyl groups are more likely to be oxidized to form lipid peroxides $(\mathrm{L}-\mathrm{OOH})$ compared with saturated fatty acids (SFA) and monounsaturated fatty acids (MUFA).[9] Therefore, the changes of metabolic feature in ccRCC makes it inherently prone to a new form of regulated cell death, ferroptosis, which is characterized by the accumulation of iron-dependent reactive oxygen species (ROS) and LPO to lethal levels.[7, 8, 10, 11] Briefly, PUFAs in cells are more likely to be oxidized by ROS (e.g., hydroxyl radical ('OH)) to form L$\mathrm{OOH}$. Then, the $\mathrm{L}-\mathrm{OOH}$ react with redox-active transition metals such as ferrous iron $\left(\mathrm{Fe}^{2+}\right)$ to produce reactive accumulation of alkoxyl radicals $\left(\mathrm{L}-\mathrm{O}^{\circ}\right)$ that can deplete nucleic acids and proteins. Eventually, the extensive peroxidation of lipids including $\mathrm{L}-\mathrm{OOH}$ and $\mathrm{L}-\mathrm{O}^{\circ}$ results in bio-membrane destabilization and micelle formation, driving cells to undergo ferroptosis.[12-15]

In recent years, numerous studies have focused on the development of iron-based nanomaterials that catalyze ${ }^{\circ} \mathrm{OH}$ generation by the Fenton reaction between $\mathrm{H}_{2} \mathrm{O}_{2}$ and $\mathrm{Fe}^{3+} / \mathrm{Fe}^{2+}$ to attack PUFAs and initiate ferroptosis.[16-18] However, the intratumoral $\mathrm{H}_{2} \mathrm{O}_{2}$ concentration $\left(50-100 \times 10^{-6} \mathrm{M}\right)$ is limited and insufficient to produce desirable and enough ${ }^{\circ} \mathrm{OH}$ for inducing satisfactory nanocatalytic-therapeutic efficacy via Fenton or Fenton-like reactions.[19] In addition, tumor cells have evolved powerful antioxidant systems to prevent lipid peroxidation and ferroptosis, resulting in limited antitumor efficiency when relying solely on Fenton reaction. Reduced glutathione (GSH), as a vital antioxidant molecule, can cycle between oxidized glutathione (GSSG) by participating in redox biochemical reactions, enabling GSH/GSSG to be an essential antioxidant defense conserved in eukaryotes.[20, 21] Cysteine is a building block for the biosynthesis of $\mathrm{GSH}$, and system $\mathrm{x}_{\mathrm{c}^{-}}$, as a transmembrane cystine-glutamate antiporter, imports cystine into cells, which plays a key role in GSH synthesis and cellular redox regulation.[22, 23] On the other hand, glutathione peroxidase 4 (GPX4), as a major antioxidant enzyme can directly reduce lipid hydroperoxides to form lipid alcohols by using two GSH molecules as electron donors, which prevents the transition metal (e.g., $\mathrm{Fe}^{2+}$ )-dependent formation of toxic lipid reactive oxygen species. [9] Therefore, to induce ferroptosis using iron-based nanomaterials, the Fe dose needs to be very high, even up to $75 \mathrm{mg} \mathrm{kg}^{-1}$ body weight, via intravenous injection,[17] and most of the reported iron-based nanomaterials need to either include an additional component that can contribute to ferroptosis, or be combined with other therapeutic approaches (e.g., chemotherapy).[19, 24] It is worth noting that erastin, sulfasalazine, ras-selective lethal small molecules (RSL3) etc. have been found to trigger accumulation of lipid hydroperoxides and ferroptosis by blocking the synthesis of GSH or directly inhibiting GPX4 activity. These small molecules have emerged as the most potent and selective compounds for killing ccRCC cells. $[8,25]$ However, their low specificity for tumors, short half-life in the blood, poor water solubility and deficient accumulation at the tumor site prevents their reliable application in vivo. Nanotechnology has been widely investigated to develop advanced drug delivery system for cancer therapy, with the intrinsic 
physicochemical properties of nanomaterials. Metal-organic frameworks (MOFs) with a high loading capacity attributed to their large surface area and ultra-high porosity enable them to be promising platforms for hydrophobic small molecule drugs delivery.[26] Particularly, Iron based MOFs nanoparticles (MIL-101(Fe) NPs) with great biocompatibility and degradability provides the possibility of achieving stimuli-responsive controlled release of various pharmacological molecules.[27, 28]

In this study, MIL-101(Fe) nanoparticles are loaded with RSL3 to form a new ferroptosis agonist (MIL101(Fe)@RSL3) in which MIL-101(Fe) released RSL3 and ferric ions $\left(\mathrm{Fe}^{3+}\right)$ accompanying with the degradation in the acidic tumor environment. $\mathrm{Fe}^{3+}$, as a degradation product, can be reduced to ferrous ions $\left(\mathrm{Fe}^{2+}\right)$ by ferric reductase and other reductive molecules in the cell, thereby triggering "iron overload". [29] $\mathrm{Fe}^{2+}$ catalyzes the intrinsic nontoxic $\mathrm{H}_{2} \mathrm{O}_{2}$ to form highly reactive ${ }^{\circ} \mathrm{OH}$ by the Fenton reaction in cancer cells, which attacks PUFAs to form $\mathrm{L}-\mathrm{OOH}$ and initiates the ferroptosis.[27] Meanwhile, RSL3 directly inhibits GPX4 to further induce the aberrated accumulation of $\mathrm{L}-\mathrm{OOH}$, triggering severe ferroptosis. Subsequently, $\mathrm{Fe}^{2+}$ continues to catalyze the generation of highly reactive lipid alkoxyl radicals $\left(\mathrm{L}-\mathrm{O}^{*}\right)$ from $\mathrm{L}-\mathrm{OOH}$, thereby aggravating ferroptosis-based cell death (Scheme 1). Therefore, MIL$101(\mathrm{Fe})$ is not only an RSL3 delivery platform for improving tumor specificity and prolonging the circulation half-life but also a ferric ion supplier for increasing the labile iron pool. MIL-101(Fe)@RSL3 is expected to provide a promising nanodrug for clear-cell renal cell carcinoma therapy by triggering waterfall-like ferroptosis based on "iron overload" and the antioxidative system suppression.

\section{Materials And Methods}

Materials

PEG-COOH (MW: 2000), 2-aminoterephthalic acid $\left(\mathrm{H}_{2} \mathrm{BDC}-\mathrm{NH}_{2}\right)$, chloroform and $\mathrm{FeCl}_{3} \cdot 6 \mathrm{H}_{2} \mathrm{O}$ were purchased from Aladdin Reagent (Shanghai, China). RSL3 was obtained from Meilun Biotechnology Co., Ltd. (Dalian, China). Cell Counting Kit-8 (CCK-8), Prussian Blue iron stain kit, and Calcein-AM/propidium iodide (PI) double stain kit was obtained from Solarbio Science \& Technology Co, Ltd. (Beijing, China). LysoTracker Red, a cellular glutathione peroxidase assay kit, and 2',7'-dichlorofluorescin diacetate (DCFDA) were purchased from Beyotime Biotechnology Co. (Beijing, China). ICG-NHS was purchased from Xi'an Ruixi Biotechnology Co., Ltd. (Xi'an, China). The anti-GPX4 and $\beta$-actin antibodies were purchased from Abcam (CA, USA). BODIPY581/591-C11 was obtained from Thermo Fisher (Waltham, MA, USA).

Instruments

The morphology and elemental mapping images were recorded with a transmission electron microscope (FEI Tecnai F20, acceleration voltage $=200 \mathrm{kV}$ ). The hydrodynamic sizes and zeta potentials of the NPs were detected with a Malvern Zetasizer Nano ZS (Malvern, UK). Confocal microscopy images were obtained with a confocal laser scanning microscope (Leica TCS SP8 STED, Germany), and the flow cytometry assay was performed with a flow cytometer (BD FACSAria ${ }^{\mathrm{TM}}$ III, USA). In vivo imaging was performed with an IVIS imaging system (PerkinElmer, USA). 
The synthetic procedure of MIL-101(Fe) NPs was consistent with a previous report.[30] Briefly, $2.48 \mathrm{mmol}$ $(0.4492 \mathrm{~g})$ of $\mathrm{H}_{2} \mathrm{BDC}-\mathrm{NH}_{2}$ and $5.0 \mathrm{mmol}(1.35 \mathrm{~g})$ of $\mathrm{FeCl}_{3} \cdot 6 \mathrm{H}_{2} \mathrm{O}$ were dissolved in $60 \mathrm{~mL}$ of DMF, and the mixture was packaged in a Teflon-lined stainless-steel autoclave and kept at $120^{\circ} \mathrm{C}$ for 12 hours. After cooling to room temperature, the precipitate was obtained by centrifugation and washed with ethanol three times.

Drug loading and surface modification

MIL-101(Fe) NPs (25 mg) were dispersed into $10 \mathrm{~mL}$ of chloroform, and $1.5 \mathrm{mg}$ of RSL3 was added to the solution under ultrasonication for $30 \mathrm{~min}$. Then, the reaction system was continuously stirred overnight. After solvent evaporation, the obtained NPs were suspended in deionized water and dialyzed against distilled water to obtain the MIL-101(Fe)@RSL3 nanocomposites. PEG-COOH was used for the surface modification of MIL-101(Fe)@RSL3 nanocomposites. First, 20 mg of PEG-COOH (MW: 2000) was dissolved in water and $2 \mathrm{mg}$ of 1-ethyl-3-(3-dimethylaminopropyl) carbodiimide (EDC) and $1.5 \mathrm{mg}$ of NHS were added to react for 4 hours, and then the mixed solution was stirred with $25 \mathrm{mg}$ of the MIL101(Fe)@RSL3 nanocomposites for 16 hours. The PEGylated MIL-101(Fe)@RSL3 NPs were recycled by centrifugation ( $8500 \mathrm{rpm}, 10 \mathrm{~min})$, and the supernatant was collected to determine the content and release of RSL3 by HPLC.

Analysis of drug and iron ions releasing in vitro

The release profiles of RSL3 and iron ions $\left(\mathrm{Fe}^{3+}\right)$ were analyzed according to previous reports.[15, 31] PEG-MIL-101(Fe)@RSL3 NPs were dispersed in PBS at pH 7.4 and pH 5.0 containing 5\% (w/v) sodium dodecyl sulfate for different durations. The mixtures were encapsulated in the regenerated cellulose membrane (MWCO: $1000 \mathrm{Da}$ ), and the buffer was stirred with a magnetic flea. At pre-determined time points, buffer was withdrawn for drug concentration analysis by HPLC. For Fe ${ }^{3+}$ analysis, PEG-MIL101(Fe)@RSL3 NPs (1 mg/mL) were re-suspended in PBS at pH 7.4 or 5.0. Then, the Fe ${ }^{3+}$ concentration was spectrophotometrically calculated in the o-phenanthroline reaction system at different times.

Cell and animal model

All cell lines were purchased from the Cell Bank of Type Culture Collection of the Chinese Academy of Sciences (Shanghai, China). Human renal clear cell adenocarcinoma cell line 786-0 cells were cultured in RPMI-1640 (Gibco, USA) media; human renal tubular epithelial cell line HK-2 and human hepatocyte cell line LO2 cells were cultured in DMEM (Gibco, USA) media. All media were supplemented with 10\% fetal bovine serum (Gibco, USA) and 1\% penicillin/streptomycin. All cells were cultured in a humidified incubator at $37^{\circ} \mathrm{C}$ with $5 \% \mathrm{CO}_{2}$.

Female BALB/C nude mice were sourced from Guangdong Medical Laboratory Animal Center (Guangdong, China) and used according to the protocols approved by the Animal Ethics Committee of 
Jinan University. The tumor model was established by subcutaneously injecting $4 \times 10^{6} 786-0$ cells suspended in PBS ( $\mathrm{pH} 7.4)$ into the dorsum of each mouse. When the tumor volumes reach to $50 \mathrm{~mm}^{3}$, the mice were applied for experiment.

Cellular uptake and subcellular localization in vitro

786-0 cells were seeded in 6-well culture plates for 24 hours. Then, the ICG-labelled MIL-101(Fe)@RSL3 NPs were incubated with the cells for 1, 2, 4, and 8 hours, and the cell culture medium was removed. After washing with PBS twice, the cells were collected and re-suspended in $500 \mu \mathrm{L}$ of PBS. Then, the cells of different groups were evaluated by flow cytometry.

To evaluate the subcellular localization of MIL-101(Fe) NPs, 786-0 cells were seeded in $20 \mathrm{~mm}$ glassbottom cell culture dishes. After removing the cell culture medium, Lyso Tracker Red staining solution was incubated with cells for 20 min. Then, ICG-labelled MIL-101(Fe)@RSL3 was added followed by incubation for an additional 1 hour. The cells were observed by CLSM (Ex/Em: 543 nm/590 nm for LysoTracker Red and Ex/Em: 633 nm/780 nm for MIL-101(Fe)@RSL3-ICG).

Intracellular iron ion release and ROS detection

786-0 cells were seeded in $20 \mathrm{~mm}$ glass-bottom cell culture dishes and incubated for 24 hours. Then, PBS, MIL-101(Fe), free RSL3 or MIL-101(Fe)@RSL3 (RSL3 dose: $1.4 \mu \mathrm{M})$ was added to the cells followed by incubation for another 3 hours. After that, the cells were washed with Hanks' balanced salt solution (HBSS) and incubated in HBSS containing Phen Green SK $(20 \mu \mathrm{M})$ for $20 \mathrm{~min}$. The cells were washed with HBSS before CLSM observation (Ex/Em: $488 \mathrm{~nm} / 517 \mathrm{~nm}$ ).

Intracellular ROS was determined by using DCFH-DA. 786-0 cells were treated with different samples, and then DCFH-DA $(10 \mu \mathrm{M})$ in serum-free RPMI 1640 medium was added. After coincubation for 30 min, the cells were washed with PBS before CLSM observation (Ex/Em: 488 nm/529 nm).

Lipid peroxide detection

Intracellular LPO was detected using BODIPY581/591-C11, which can insert into lipid membranes and be oxidized by LPO. 786-0 cells were seeded in $20 \mathrm{~mm}$ glass-bottom cell culture dishes and incubated for 24 hours. Then, PBS, MIL-101(Fe), free RSL3 or MIL-101(Fe)@RSL3 (RSL3 dose: 1.4 $\mu \mathrm{M}$ ) was added to the cells followed by incubation for another 3 hours. After that, the cells were washed with PBS and incubated in serum-free RPMI 1640 medium containing BODIPY581/591-C11 (5 $\mu$ M) for 20 min. After washing with PBS, the cells were subjected to CLSM observation. The oxidized products display green fluorescence (Ex/Em: $485 \mathrm{~nm} / 520 \mathrm{~nm}$ ).

GPX4 activity and level assay

GPX4 activity was measured with a commercial glutathione peroxidase assay kit. 786-0 cells were treated with PBS, MIL-101(Fe), free RSL3 or MIL-101(Fe)@RSL3 (RSL3 dose: 1.4 $\mu \mathrm{M})$ ). Twelve hours later, 
the cells were harvested in PBS solution containing $0.2 \%(\mathrm{w} / \mathrm{v})$ ethylenediaminetetraacetic acid (EDTA). Then, GPX4 activity was detected according to the manufacturer's protocol. The absorbance at $340 \mathrm{~nm}$ was recorded every 1 min with a SpectraMax microplate reader with the temperature maintained at $25{ }^{\circ} \mathrm{C}$.

We also employed a Western blotting approach to assess GPX4 levels. In brief, the lysate of formulationtreated 786-0 cells was collected for analysis. The level of protein in these samples was quantified. Then, samples containing equal amounts of protein $(20 \mu \mathrm{g})$ were loaded and subjected to standard SDS polyacrylamide gel electrophoresis. The proteins were separated at a constant voltage, followed by electrical transfer to a $0.45 \mu \mathrm{m}$ poly (vinylidene fluoride) (PVDF) membrane. Upon membrane blocking with $5 \%$ milk phosphate buffered saline with Tween 20 (PBST) solution for 1 hour at room temperature, the target proteins were incubated with GPX4-specific antibody and $\beta$-actin antibody overnight at dilutions of 1:2500 and 1:5000, respectively, at $4{ }^{\circ} \mathrm{C}$. Thereafter, HRP-conjugated anti-rabbit IgG H\&L at a dilution of 1:5000 was applied as the secondary antibody, and the specific bands were developed using ECLTM Western blotting detection reagents.

Cytotoxic and anti-tumor effect in vivo

The cytotoxicity measurement was quantified according to the standard CCK-8 approach. 786-0 cells were seeded in 96 -well plates at a density of $5 \times 10^{3}$ cells/well and incubated for 24 hours. Then, MIL101(Fe), free RSL3 or MIL-101(Fe)@RSL3 was added to each well at different concentrations. After 24 hours of incubation, CCK-8 solution was added to each well, and the cells were incubated for an additional 1 hour. Afterwards, the absorbance was measured with a microplate reader at $450 \mathrm{~nm}$. Cell viability was calculated by the ratio of the absorbance of the sample to the absorbance of the control, and each test was repeated at least three times. Then, calcein-AM and PI were used for live/dead cell staining after different treatments.

In vivo fluorescence imaging and biodistribution of nanodrugs

To track the biodistribution of MIL-101(Fe)@RSL3, ICG-labelled MIL-101(Fe)@RSL3 was intravenously injected into mice. Fluorescence images of mice were recorded at different time points with an IVIS imaging system, and then the tumor and main organs were harvested for fluorescence imaging. Blood circulation was measured by drawing $10 \mu \mathrm{L}$ of blood from the tail vein of the tumor-bearing mice at different times after intravenous injection of ICG-labelled PEG-MIL-101(Fe)@RSL3. Each blood sample was dissolved in $140 \mu \mathrm{L}$ of lysis buffer ( $1 \%$ sodium dodecyl sulfonate, $1 \%$ Triton $\mathrm{X}-100,40 \times 10^{-3} \mathrm{M}$ Tris acetate), and the fluorescence intensities were measured using a microplate reader (excitation wavelength: $789 \mathrm{~nm}$, emission wavelength: $814 \mathrm{~nm}, \mathrm{n}=3$ ). The blank blood sample was also measured as the control to subtract blood autofluorescence.

Side effects evaluation

Human normal hepatocyte LO2 and human renal tubular epithelial cell HK-2 cells were seeded into 96well plates and treated with a series of MIL-101(Fe)@RSL3 concentrations (RSL3 dose 0, 0.35, 0.70, 1.40, 
$2.80,5.60 \mu \mathrm{M})$. Cell viability was evaluated by CCK-8 assay, which was conducted according to the manufacturer's protocol.

Whole blood was collected from the mice and used for haemolytic analysis. After centrifugation at 800 rpm, the upper serum was discarded, and the lower blood cells were washed with cold PBS three times and mixed with a certain amount of MIL-101(Fe)@RSL3. Twenty-four hours later, the mixture was centrifuged at $800 \mathrm{rpm}$, and the absorbance of the supernatant was measured at $350 \mathrm{~nm}$ to calculate the haemolysis rate.

To evaluate side effects in vivo, the mice were sacrificed seven days after treatment. Approximately 0.8 $\mathrm{mL}$ of blood was collected from each mouse to conduct complete blood panel analysis and serum biochemistry assays at the Laboratory of Comparative Medicine, Guangdong Medical Laboratory Animal Center. The major organs of the mice from each group were collected and stained with H\&E according to standard techniques to evaluate toxicity.

Anti-tumor effect in vivo

BALB/c nude mice were randomly divided into four groups $(n=6)$. The nanodrugs were intravenously injected into mice three times a week for a total of 5 injections. The tumor volume was measured every three days according to the formula: $V=\left(L \times W^{2}\right) / 2$, where $L$ is the tumor length and $W$ is the tumor width. The relative tumor volume was calculated according to the equation: $\mathrm{V} / \mathrm{V}_{0}$, where $\mathrm{V}_{0}$ is the initial tumor volume before the start of treatment. Then, 21 days later, the tumor tissues were excised for weighing and histological examination.

Statistical analysis

The data are presented as the mean \pm standard deviation (SD). The difference of each treatment group was statistically compared via either Student's t test or analysis of variance integrated with Tukey's post hoc analysis. The threshold $P$ value was set at 0.05 .

\section{Results}

Characterization of MIL-101(Fe)@RSL3 nanoparticles (NPs)

Iron-based MOF NPs, MIL-101(Fe), were prepared by the solvothermal method with simple materials $\left(\mathrm{FeCl}_{3} \cdot 6 \mathrm{H}_{2} \mathrm{O}\right.$ and 2-aminoterephthalic acid). The MIL-101(Fe) morphology was visualized by transmission electron microscopy (TEM) and the particles showed good dispersibility and a uniform size of approximately $150 \mathrm{~nm}$ (Figure 1A). After modification with polyethylene glycol (PEG)-COOH, a nearly transparent thin layer of PEG appeared on the surface of MIL-101(Fe) (Figure 1B). Additionally, the average hydrodynamic diameter of MIL-101(Fe) changed from $174 \mathrm{~nm}$ to $190 \mathrm{~nm}$ (Figure 1C), and the zeta potential reversed from $34.5 \pm 0.6$ to $-23.7 \pm 0.5$ after modification (Figure 1D), which was measured by dynamic light scattering (DLS). The increased size and reversed surface charge suggested that MIL- 
101(Fe) had been successfully modified with PEG. It is well known that MOF NPs possess tunable porous structures and high specific surface areas, which facilitate drug loading and release. Herein, a hydrophobic small molecular (1S,3R-RSL3) was loaded into MIL-101(Fe) to form a drug delivery composite (MIL-101(Fe)@RSL3). Energy-dispersive X-ray spectroscopy (EDX) elemental mapping of MIL101(Fe)@RSL3 showed C, O, Fe and Cl, which confirmed that RSL3 was loaded into MIL-101(Fe) (Figure 1E). Fourier transform infrared spectroscopy (FTIR) was used to analyze the PEGylated MIL101(Fe)@RSL3 (Figure S1). The vibrational band at approximately $585 \mathrm{~cm}^{-1}$ belonged to the Fe-0 vibration, and the peaks at $3363 \mathrm{~cm}^{-1}$ and $3466 \mathrm{~cm}^{-1}$ were ascribed to the asymmetrical and symmetrical stretching vibrations of the amine groups, respectively.[32] After loading with RSL3, the characteristic C-Cl peak at $742 \mathrm{~cm}^{-1}$ appeared in PEG-MIL-101(Fe)@RSL3. Additionally, the band at $2883 \mathrm{~cm}^{-1}$ attributed to $\mathrm{OH}$ disappeared, and the bands from the amine groups at $3466 \mathrm{~cm}^{-1}$ weakened, suggesting the formation of amide bonds between the - $\mathrm{COOH}$ of PEG and the $-\mathrm{NH}_{2}$ of MIL-101(Fe). Hereafter, these PEGylated nanoparticles were abbreviated as MIL-101(Fe) and MIL-101(Fe)@RSL3. The RSL3 content in MIL$101(\mathrm{Fe})$ was further analyzed by HPLC, and the loading efficiency was $2.23 \%$ with an encapsulation efficiency of $88.7 \%$, indicating a highly efficient drug loading properties. Apart from their drug delivery capability, MIL-101(Fe) NPs showed a pH-dependent drug release profile in vitro (Figure 1F), and the RSL3 release rate in the simulated lysosomal microenvironment ( $\mathrm{pH} 5.0)$ was much faster than that in neutral phosphate buffer ( $\mathrm{pH}$ 7.4). The MIL-101(Fe) NPs remained stable in $\mathrm{pH} 7.4$ solution even after incubation for 12 hours (Figure S2A). In contrast, when exposed to acidic conditions (pH 5.0), the structure of MIL101(Fe) NPs showed a tendency to degrade and collapse (Figure S2B), which was consistent with previous reports.[27, 31] Moreover, whether the MIL-101(Fe) NPs could release iron ions were colourimetrically determined with the products of o-phenanthroline and ferrous ions. After incubation with MIL-101(Fe) NPs in PBS pH 7.4 (Figure S3C) and pH 5.0 (Figure S3D) for 1, 2, 4, 8, 12, and 24 hours, the supernatants became reddish-orange in color, which was attributed to the iron ion release into solution. It could be clearly observed that the number of iron ions released into the acidic solution increased significantly. According to calculations from the standard curve (Figure S3A, B), the iron ion concentrations in the supernatants were $0.21 \mathrm{mM}$ at pH 7.4 and $0.50 \mathrm{mM}$ at pH 5.0 (Figure 1G). Hydroxyl radicals $\left({ }^{\circ} \mathrm{OH}\right)$ were generally considered to be the most toxic ROS, which could be generated by the Fenton reaction between $\mathrm{H}_{2} \mathrm{O}_{2}$ and $\mathrm{Fe}^{3+} / \mathrm{Fe}^{2+}$ to result in the lethal lipid peroxidation and initiate ferroptosis. [23, 33] To investigate whether MIL-101(Fe) NPs could produce ${ }^{\circ} \mathrm{OH}$ in the presence of $\mathrm{H}_{2} \mathrm{O}_{2}$, 5,5-dimethyl-1-pyrroline $\mathrm{N}$-oxide (DMPO) was employed as a trapping probe to monitor the ${ }^{\circ} \mathrm{OH}$ generation by electron paramagnetic resonance (ESR). It was found that the characteristic 1:2:2:1 signal in the ESR spectrum attributing to ${ }^{\circ} \mathrm{OH}$ was observed for the MIL-101(Fe) NPs incubated with $\mathrm{H}_{2} \mathrm{O}_{2}(1 \mathrm{mM})$ at acidic (pH 5.0) and neutral ( $\mathrm{pH7.4)}$ solution (Figure $1 \mathrm{H}$ ). Particularly, the stronger ${ }^{\circ} \mathrm{OH}$ signals were observed at acidic solution, indicating the acidic microenvironment promoted the release of iron ions and thus increased the production of ${ }^{\circ} \mathrm{OH}$. Moreover, the production of ${ }^{\circ} \mathrm{OH}$ was further detected by methylene blue (MB) decolorization experiments. More obvious color fading of $\mathrm{MB}$ was observed in acidic solution after mixed with MIL-101(Fe) and $\mathrm{H}_{2} \mathrm{O}_{2}$ (Figure S4A) than neutral solution (Figure S4B), which further 
confirmed the acidic environment accelerated the ${ }^{\circ} \mathrm{OH}$-production. Overall, with excellent biocompatibility and biodegradable properties, MIL-101(Fe) NPs serve as an ideal drug delivery platform and continuously provide iron ions for ${ }^{\circ} \mathrm{OH}$-production through the Fenton reaction.

Intracellular iron ion release and ferroptosis of ccRCC cells.

To further investigate the intracellular iron ion released from MIL-101(Fe)@RSL3, the NPs were prelabelled with indocyanine green (ICG) on the surface and the cellular uptake was evaluated by fluorescence-activated cell sorting (FACS). After the MIL-101(Fe)@RSL3-ICG NPs were incubated with human RCC cells (786-O cells), the fluorescence intensity of ICG was significantly enhanced with prolonged incubation time, which indicated the cellular accumulation of fluorescently labelled NPs (Figure S5). Then, the intracellular localization of MIL-101(Fe)@RSL3-ICG NPs was determined by confocal laser scanning microscopy (CLSM). Notably, the fluorescence of MIL-101(Fe)@RSL3-ICG matched that of LysoTracker Red (Figure 2A), which suggested that MIL-101(Fe)@RSL3-ICG was internalized by endosomes or lysosomes. The extracellular microenvironment of solid tumors is acidic $(\mathrm{pH}$ 6.5 7.0) and lower than that of normal tissues ( $\mathrm{pH} \mathrm{7.4),} \mathrm{and} \mathrm{the} \mathrm{tumor} \mathrm{endosomes/lysosomes} \mathrm{are}$ severely acidic, reaching $\mathrm{pH}$ values as low as 4.5 5.5.[34] The acidic microenvironment of endosomes/lysosomes easily triggers the degradation of MIL-101( $\mathrm{Fe})$ and the release of $\mathrm{Fe}^{3+}$ ions. Moreover, the presence of reducing agents such as ferrireductase, superoxide anion radicals $\left(\mathrm{O}_{2}{ }^{-{ }^{-}}\right)$, cysteine and glutathione can reduce the $\mathrm{Fe}^{3+}$ ions into $\mathrm{Fe}^{2+}$ ions, and then $\mathrm{Fe}^{2+}$ ions can be transported to the labile iron pool in the cytoplasm by divalent metal transporter 1 (DMT1). $[35,36]$ The intracellular Fe ${ }^{2+}$ ion fluorescent probe Phen Green SK (PGSK) was applied to detect changes in the $\mathrm{Fe}^{2+}$ ion levels. The fluorescence of PGSK was remarkably reduced in the MIL-101(Fe) and MIL-101(Fe)@RSL3 incubation groups owing to $\mathrm{Fe}^{2+}$ ion chelation (Figure 2B), which manifested the degradation of MIL-101(Fe) in the endosomes/lysosomes and enhanced the intracellular labile iron pool level. Next, to investigate whether the MIL-101( $\mathrm{Fe}$ ) would catalyze the ${ }^{\circ} \mathrm{OH}$ production via the Fenton reaction, the aminophenyl fluorescein (APF), an ${ }^{\circ} \mathrm{OH}$-specific fluorescent probe, was used to verify the intracellular ${ }^{\circ} \mathrm{OH}$ level. After incubated with MIL-101(Fe), the fluorescence of ${ }^{\circ} \mathrm{OH}$ was slightly enhanced. Interestingly, fluorescence signal was significantly enhanced in the MIL-101(Fe)@RSL3 group, suggesting a burst of ' $\mathrm{OH}$ in 786-0 cells (Figure 2C). It suggested that MIL-101( $\mathrm{Fe}$ ) could catalyze the ${ }^{\circ} \mathrm{OH}$ production via the Fenton reaction attributing to the much higher content of $\mathrm{H}_{2} \mathrm{O}_{2}$ in cancer cells (up to $\left.100 \times 10^{-6} \mathrm{M}\right)$ than in normal tissues $\left(\approx 20 \times 10^{-9}\right.$ $M)$. The $\mathrm{OH}$ would cause the peroxidation of polyunsaturated fatty acid-containing phospholipids (PUFAs) to form lipid peroxides $(\mathrm{L}-\mathrm{OOH})$ and propagate the radical process to adjacent lipid molecules. $[22,37]$ Although there had been reported that an increase in the labile iron pool caused by intracellular iron overload would trigger ferroptosis, cellular iron overload was not sufficient to produce enough intracellular ${ }^{\circ} \mathrm{OH}$ by solely depending on the Fenton reaction. $[17,38]$ The strong antioxidant system in cells would block the propagation process of $\mathrm{L}-\mathrm{OOH}$, and prevented the generation of highly reactive lipid alkoxyl radicals $(\mathrm{L}-\mathrm{O} \cdot)$ via Fenton chemistry between $\mathrm{Fe}^{2+}$ and $\mathrm{L}-\mathrm{OOH} .[9,13]$ Therefore, it was 
expected to be an effective way to induce ferroptosis by producing the ${ }^{\circ} \mathrm{OH}$ and inhibiting the antioxidant system to disrupt the redox balance of cells.

As an antioxidant enzyme, GPX4 is essential to maintain lipid homeostasis in cells, preventing the accumulation of lipid peroxidation and blocking ferroptosis. RSL3, a direct inhibitor of GPX4, was expected to be a small molecule drug that induces $\mathrm{L}-\mathrm{OOH}$ in tumors, but its insolubility and poor pharmacokinetic properties have limited its application. Fortunately, in addition to providing iron ions to cancer cells, MIL-101(Fe) NPs can also serve as an RSL3 delivery platform. To evaluate the inhibition efficiency of RSL3, nicotinamide adenine dinucleotide phosphate (NADPH) levels, which indirectly reflect GPX4 activity, were detected. MIL-101(Fe) did not cause a loss of activity of GPX4, showing activity analogous to that of the control group. In contrast, intracellular NADPH remained almost constant in the free RSL3 and MIL-101(Fe)@RSL3 treatment groups, indicating a decrease in GPX4 activity (Figure 3A). Moreover, GPX4 expression showed a remarkable decrease after RSL3 and MIL-101(Fe)@RSL3 treatment (Figure 3B), which can be easily explained by the mechanism of action of RSL3 as a powerful GPX4 inhibitor. This result implied that RSL3 could be released from MIL-101(Fe) NPs and exert an inhibitory effect. Then, BODIPY-C11, a membrane-targeted fluorescence probe of $\mathrm{L}-\mathrm{OOH}$, was used for staining the various treatment groups.[39] MIL-101(Fe) NPs only slightly increased the oxidized BODIPY-C11 signal, whereas RSL3 and MIL-101(Fe)@RSL3 strongly enhanced the fluorescence of oxidized BODIPY-C11 (Figure $3 \mathrm{C}$ ), indicating a breakdown of the redox equilibrium. In addition, the intracellular oxidative stress of 786-0 cells after various treatments was carefully studied by utilizing DCFH-DA as the intracellular total ROS probe. Both the fluorescence images (Figure S6A) and quantitatively analyst results (Figure S6B) showed that MIL-101(Fe)@RSL3 triggered ROS burst and accumulation, which was owing to the iron overload and the GPX4 inhibition. Therefore, the accumulation of lipid ROS induced by MIL101(Fe)@RSL3 is expected to trigger more violent ferroptosis.

Cell viability assays indicated that MIL-101(Fe)@RSL3 showed significant cytotoxicity against ccRCC cells compared to RSL3 treatment. Even at a low concentration of loaded RSL3 (1.4 $\mu \mathrm{M})$, the cell survival rate in the MIL-101(Fe)@RSL3 group was only 28\% (Figure 3D). In contrast, the high concentration of MIL-101(Fe) NPs showed negligible cytotoxicity. Furthermore, live/dead cell fluorescence staining showed that MIL-101(Fe)@RSL3 induced evident cytotoxicity compared with the other treatment groups (Figure 3E). However, normal cells, such as human hepatocyte cells (LO2) and human renal proximal tubular cells (HK-2), maintained a high cellular survival rate even at 11.2 $\mu \mathrm{M}$ MIL-101(Fe)@RSL3 NPs, which was 8 times higher than the concentration for ccRCC treatment (Figure S7). Interestingly, MIL-101(Fe)@RSL3 did not cause severe cytotoxicity in normal human hepatocytes or renal cells, and it is well known that the liver and kidney are vital organs that metabolize NPs.

In vivo antitumor efficiency of MIL-101(Fe)@RSL3 against ccRCC

Good compatibility is a prerequisite for the clinical application of nanodrugs. The negligible cytotoxicity of MIL-101(Fe)@RSL3 in normal cells inspired us to further investigate the antitumor effects of MIL101(Fe)@RSL3 in ccRCC. As we expected, after incubation with a high concentration of MIL- 
101(Fe)@RSL3 (2 mg/mL) for 24 hours, the haemolysis rate was only $0.5 \%$, which can almost be ignored (Figure S8). Then, the nanodrugs were labelled with ICG to analyze their tissue distribution and tumor accumulation in vivo. After intravenous injection of ICG-MIL-101(Fe)@RSL3, the whole bodies of tumorbearing mice showed bright fluorescence, and the tumor region showed an obvious fluorescence enhancement (Figure 4A). This result suggested that ICG-MIL-101(Fe)@RSL3 accumulated to a great extent in tumors, especially one-hour post-injection. Subsequently, the fluorescence gradually faded away; however, the tumor region remained bright up to 12 hours later (Figure 4B). The blood circulation behavior of ICG-MIL-101(Fe)@RSL3 was determined by evaluating the ICG fluorescence from the blood samples collected via the tail vein at various time points, and the half-life of MIL-101(Fe)@RSL3 was calculated to be $~ 2.5$ hours (Figure 4C). Then, the main organs and tumor were isolated for fluorescence imaging. Similar to the fluorescence imaging in vivo, the fluorescence intensity of the tumor was significantly higher than that of the heart, lung, etc. (Figure 4D), while the high-intensity fluorescence in the liver, intestine, etc. suggested that the MIL-101(Fe)@RSL3 NPs would be cleared by the liver and finally excreted in bile and faeces (Figure 4E).

Antitumor effects from the MIL-101(Fe)@RSL3 NPs were detected after intravenous administration. Tumor growth was constantly recorded over 21 days (Figure S9), and the changes in the tumor volumes indicated that MIL-101(Fe)@RSL3 remarkably inhibited tumor growth (Figure 5A). It is worth noting that the RSL3 treatment group showed only limited tumor suppression, which can probably be attributed to the insolubility of RSL3 in water and its deficient accumulation at the tumor site (Figure 5B, C). Moreover, haematoxylin and eosin (H\&E) and Ki-67 staining of tumor tissue sections from the MIL-101(Fe)@RSL3 group also showed significantly fewer cancer cells and abundant karyorrhectic debris than the other groups, indicating more obvious damage to the cancer cells (Figure 5D). Although both the MIL-101(Fe) and MIL-101(Fe)@RSL3 treatment groups showed extensive blue spots from Prussian blue staining, indicating the accumulation, degradation and release of iron ions from MIL-101(Fe) NPs into the tumors, the MIL-101(Fe) NPs still did not show obvious antitumor effects (Figure S10). In RSL3 and MIL101(Fe)@RSL3 groups, the immunohistochemical staining of tumor sections showed the level of GPX4 decrease, suggesting the RSL3 released from MIL-101(Fe) and inhibited the GPX4 (Figure 5E). These results suggested that solely causing iron overload proved to be unsuccessful in triggering severe ferroptosis and unable to have a satisfactory antitumor effect because of the powerful intracellular antioxidant network. Synchronous destroying the antioxidant network and causing iron overload could remarkably inhibit tumor growth by aggravating ferroptosis, while simply inhibiting the antioxidant network or triggering iron overloading showed only moderate or negligible antitumor effects in vivo.

Side effects of MIL-101(Fe)@RSL3

The potential side effects of the MIL-101(Fe)@RSL3 nanoagents were evaluated after systemic administration. Haematological and blood biochemical analyses were performed one day post-treatment. Standard haematology markers, including white blood cells (WBCs), red blood cells (RBCs), haemoglobin (HGB), mean corpuscular volume (MCV), mean corpuscular haemoglobin (MCH), mean corpuscular haemoglobin concentration (MCHC), platelets (PLTs) and haematocrit (HCT), were measured (Figure 6A). 
Compared with the control group, all parameters in the treatment groups appeared to be normal and did not show a significant difference ( $P$ value $>0.05)$. This result indicated that neither RSL3 nor MIL-101(Fe) NPs caused obvious inflammation in mice. In addition, blood biochemical analyses were carried out, and various parameters, including alanine transaminase (ALT), aspartate transaminase (AST), creatinine (CRE) and blood urea nitrogen (BUN), were examined (Figure 6B). There was no meaningful difference between the groups, suggesting that MIL-101(Fe)@RSL3 did not cause obvious hepatic or kidney toxicity to mice. During the treatment process, the body weights of the mice stably increased (Figure 6C). Then, histological analysis of the major organs, including the heart, liver, spleen, lung, and kidney, was conducting by slicing for H\&E staining after 21 days (Figure 6D). No apparent histological abnormalities or lesions could be observed in any of the treatment groups throughout the whole treatment period. Especially, there was no noticeable damage in the liver or kidneys, which confirmed the biocompatibility of MIL-101(Fe)@RSL3.

\section{Discussion}

It was well known that cancer cells have a higher demand for iron than the normal cells to support their rapid proliferation.[40,41] In addition, most of cancer cells showed upregulated expression of transferrin receptor 1 and hepcidin along with downregulated expression of ferroportin, enabling cancer cells to accumulate iron highly efficiently via transferrin receptors.[42, 43] However, the intracellular concentration of iron ions still lies at a low level, making it insufficient to induce ferroptosis by catalyzing the Fenton reaction due to the massive demand of rapid growth cancer cells.[15, 31] In this study, iron-based MOFs nanoparticles with $\mathrm{pH}$-responsive degradation properties could be uptake via endocytosis and release $\mathrm{Fe}^{3+}$ ions in lysosomes for remarkably increasing the intracellular content of $\mathrm{Fe}^{3+}$ ions. Subsequently, the intracellular $\mathrm{Fe}^{2+}$ referred to as the labile iron pool (LIP) also increase accordingly, because $\mathrm{Fe}^{3+}$ ions reduced to $\mathrm{Fe}^{2+}$ by the intracellular iron reductase (e.g., STEAP3) and superoxide.[44, 45] However, the intracellular ${ }^{\circ} \mathrm{OH}$ produced by $\mathrm{Fe}^{2+}$ together with $\mathrm{H}_{2} \mathrm{O}_{2}$ still remained low level only administrated with MIL-101(Fe) NPs. Even the high concentration of MIL-101(Fe) NPs (up to $106 \mu \mathrm{g} / \mathrm{mL}$ ) administration could not induce plenty of lipid peroxidation and hardly cause cell death attributed to the strong antioxidation system. On the other hand, although the RSL3 as a small molecule drug effectively triggered ferroptosis in vitro evaluation, the in vivo anti-tumor efficacy was unsatisfactory, because of the low tumor specificity, short circulating half-life in blood, poor water solubility and deficiency accumulation at tumor site. In contrast, MIL-101(Fe) NPs not only served as the drug delivery platform but also continuously provided the iron ions for cancer cells, thereby enhancing the anti-tumor effect by aggravating ferroptosis. It could not be ignored that in this study, the ferroptosis was initiated by the Fenton reaction between intracellular $\mathrm{Fe}^{2+}$ and $\mathrm{H}_{2} \mathrm{O}_{2}$, which generate ${ }^{\circ} \mathrm{OH}$ to attack PUFAs. While, the limited contents of intracellular $\mathrm{H}_{2} \mathrm{O}_{2}(100 \mu \mathrm{M} \sim 1 \mathrm{mM})$ were difficult to produce sufficient ${ }^{\circ} \mathrm{OH}$ and highly efficiently trigger ferroptosis, making it is undesirable to induce satisfactory therapeutic efficacy. Therefore, it is reasonable to believe that further elevating the intracellular oxidative stress by combining 
with chemotherapy, PDT and radiotherapy was expected to aggravate ferroptosis and achieve better curative effect.[46-48]

\section{Conclusion}

In this study, MIL-101(Fe) nanoparticles rich in iron were used to load a ferroptosis inducer (RSL3) for targeted delivery and responsive release. MIL-101(Fe) showed high encapsulation efficiency and the tumor-targeted delivery of RSL3. Under the acidic tumor microenvironment, MIL-101(Fe)@RSL3 with gradual degradation released iron ions and RSL3, thereby aggravating ferroptosis in cCRCC cells by triggering iron overload and inhibiting GPX4. Therefore, compared with free RSL3, MIL-101(Fe)@RSL3 showed more remarkable antitumor efficacy against cCRCC, which displays an abnormal PUFAs metabolic state, while these nanodrugs did not cause obvious cytotoxicity in normal cells even at very high concentrations. In addition, MIL-101(Fe)@RSL3 caused negligible side effects, enabling it to be a promising nanodrug for systemic ccRCC therapy.

\section{Declarations}

\section{Acknowledgments}

This work is supported by the National Key Research and Development Program of China (2017YFA0205200), Guangdong Basic and Applied Basic Research Foundation (2020A1515011101, 2021A1515011703, 2021A1515010697), Natural Science Foundation of Heilongjiang Province of China (LC2017038), National Natural Science Foundation of China (61805049). We would like to acknowledge the Open Fund of MOE Key Laboratory of Laser Life Science and Institute of Laser Life Science.

\section{Author contributions}

Conceptualization, Ni W., Lu C., and Wen L.; methodology, Ni W., Yuan X., Liang L.; data curation, Ni W., Liang L., Yuan X., Wen L.; writing and original draft preparation, Ni. W., Yuan X., Wen L.; writing and review and editing, Zhan M., Lu L., Xie Q., Wen L.; supervision, Lu L., Xie Q.; funding acquisition, Ni W., Lu C., Lu L., Wen L..

\section{Declarations}

The author reports no conflicts of interest in this work.

\section{References}

[1] F. Bray, J. Ferlay, I. Soerjomataram, R.L. Siegel, L.A. Torre, A. Jemal, Global cancer statistics 2018: GLOBOCAN estimates of incidence and mortality worldwide for 36 cancers in 185 countries, CA Cancer J Clin 68(6) (2018) 394-424. 
[2] T.K. Choueiri, R.J. Motzer, Systemic Therapy for Metastatic Renal-Cell Carcinoma, N Engl J Med 376(4) (2017) 354-366.

[3] A.A. Lalani, B.A. McGregor, L. Albiges, T.K. Choueiri, R. Motzer, T. Powles, C. Wood, A. Bex, Systemic Treatment of Metastatic Clear Cell Renal Cell Carcinoma in 2018: Current Paradigms, Use of Immunotherapy, and Future Directions, Eur Urol 75(1) (2019) 100-110.

[4] P.C. Barata, B.I. Rini, Treatment of renal cell carcinoma: Current status and future directions, CA Cancer J Clin 67(6) (2017) 507-524.

[5] H.I. Wettersten, O.A. Aboud, P.N. Lara, Jr., R.H. Weiss, Metabolic reprogramming in clear cell renal cell carcinoma, Nat Rev Nephrol 13(7) (2017) 410-419.

[6] I.J. Frew, H. Moch, A clearer view of the molecular complexity of clear cell renal cell carcinoma, Annu Rev Pathol 10 (2015) 263-89.

[7] H. Miess, B. Dankworth, A.M. Gouw, M. Rosenfeldt, W. Schmitz, M. Jiang, B. Saunders, M. Howell, J. Downward, D.W. Felsher, B. Peck, A. Schulze, The glutathione redox system is essential to prevent ferroptosis caused by impaired lipid metabolism in clear cell renal cell carcinoma, Oncogene 37(40) (2018) 5435-5450.

[8] Y. Zou, M.J. Palte, A.A. Deik, H. Li, J.K. Eaton, W. Wang, Y.Y. Tseng, R. Deasy, M. Kost-Alimova, V. Dancik, E.S. Leshchiner, V.S. Viswanathan, S. Signoretti, T.K. Choueiri, J.S. Boehm, B.K. Wagner, J.G. Doench, C.B. Clish, P.A. Clemons, S.L. Schreiber, A GPX4-dependent cancer cell state underlies the clearcell morphology and confers sensitivity to ferroptosis, Nat Commun 10(1) (2019) 1617.

[9] G.C. Forcina, S.J. Dixon, GPX4 at the Crossroads of Lipid Homeostasis and Ferroptosis, Proteomics 19(18) (2019) e1800311.

[10] C. Porta, L. Cosmai, B.C. Leibovich, T. Powles, M. Gallieni, A. Bex, The adjuvant treatment of kidney cancer: a multidisciplinary outlook, Nat Rev Nephrol 15(7) (2019) 423-433.

[11] R.R. Kotecha, R.J. Motzer, M.H. Voss, Towards individualized therapy for metastatic renal cell carcinoma, Nat Rev Clin Oncol 16(10) (2019) 621-633.

[12] Z. Shen, J. Song, B.C. Yung, Z. Zhou, A. Wu, X. Chen, Emerging Strategies of Cancer Therapy Based on Ferroptosis, Adv Mater 30(12) (2018) e1704007.

[13] C. Liang, X. Zhang, M. Yang, X. Dong, Recent Progress in Ferroptosis Inducers for Cancer Therapy, Adv Mater 31(51) (2019) e1904197.

[14] L. Lin, S. Wang, H. Deng, W. Yang, L. Rao, R. Tian, Y. Liu, G. Yu, Z. Zhou, J. Song, H.H. Yang, Z.Y. Chen, $X$. Chen, Endogenous Labile Iron Pool-Mediated Free Radical Generation for Cancer Chemodynamic Therapy, J Am Chem Soc 142(36) (2020) 15320-15330. 
[15] Y. An, J. Zhu, F. Liu, J. Deng, X. Meng, G. Liu, H. Wu, A. Fan, Z. Wang, Y. Zhao, Boosting the Ferroptotic Antitumor Efficacy via Site-Specific Amplification of Tailored Lipid Peroxidation, ACS Appl Mater Interfaces 11(33) (2019) 29655-29666.

[16] Z. Zhou, J. Song, R. Tian, Z. Yang, G. Yu, L. Lin, G. Zhang, W. Fan, F. Zhang, G. Niu, L. Nie, X. Chen, Activatable Singlet Oxygen Generation from Lipid Hydroperoxide Nanoparticles for Cancer Therapy, Angew Chem Int Ed Engl 56(23) (2017) 6492-6496.

[17] C. Zhang, W. Bu, D. Ni, S. Zhang, Q. Li, Z. Yao, J. Zhang, H. Yao, Z. Wang, J. Shi, Synthesis of Iron Nanometallic Glasses and Their Application in Cancer Therapy by a Localized Fenton Reaction, Angew Chem Int Ed Engl 55(6) (2016) 2101-6.

[18] F. Zhang, F. Li, G.H. Lu, W. Nie, L. Zhang, Y. Lv, W. Bao, X. Gao, W. Wei, K. Pu, H.Y. Xie, Engineering Magnetosomes for Ferroptosis/Immunomodulation Synergism in Cancer, Acs Nano 13(5) (2019) 56625673.

[19] S. Gao, H. Lin, H. Zhang, H. Yao, Y. Chen, J. Shi, Nanocatalytic Tumor Therapy by Biomimetic Dual Inorganic Nanozyme-Catalyzed Cascade Reaction, Adv Sci (Weinh) 6(3) (2019) 1801733.

[20] C. Liu, D. Wang, S. Zhang, Y. Cheng, F. Yang, Y. Xing, T. Xu, H. Dong, X. Zhang, Biodegradable Biomimic Copper/Manganese Silicate Nanospheres for Chemodynamic/Photodynamic Synergistic Therapy with Simultaneous Glutathione Depletion and Hypoxia Relief, Acs Nano 13(4) (2019) 4267-4277.

[21] S.S. Wan, Q. Cheng, X. Zeng, X.Z. Zhang, A Mn(III)-Sealed Metal-Organic Framework Nanosystem for Redox-Unlocked Tumor Theranostics, Acs Nano 13(6) (2019) 6561-6571.

[22] H. Feng, B.R. Stockwell, Unsolved mysteries: How does lipid peroxidation cause ferroptosis?, PLoS Biol 16(5) (2018) e2006203.

[23] X. Shan, S. Li, B. Sun, Q. Chen, J. Sun, Z. He, C. Luo, Ferroptosis-driven nanotherapeutics for cancer treatment, J Control Release 319 (2020) 322-332.

[24] P. Ma, H. Xiao, C. Yu, J. Liu, Z. Cheng, H. Song, X. Zhang, C. Li, J. Wang, Z. Gu, J. Lin, Enhanced Cisplatin Chemotherapy by Iron Oxide Nanocarrier-Mediated Generation of Highly Toxic Reactive Oxygen Species, Nano Lett 17(2) (2017) 928-937.

[25] W.S. Yang, R. SriRamaratnam, M.E. Welsch, K. Shimada, R. Skouta, V.S. Viswanathan, J.H. Cheah, P.A. Clemons, A.F. Shamji, C.B. Clish, L.M. Brown, A.W. Girotti, V.W. Cornish, S.L. Schreiber, B.R. Stockwell, Regulation of ferroptotic cancer cell death by GPX4, Cell 156(1-2) (2014) 317-331.

[26] M.X. Wu, Y.W. Yang, Metal-Organic Framework (MOF)-Based Drug/Cargo Delivery and Cancer Therapy, Adv Mater 29(23) (2017). 
[27] X. Ma, X. Ren, X. Guo, C. Fu, Q. Wu, L. Tan, H. Li, W. Zhang, X. Chen, H. Zhong, X. Meng, Multifunctional iron-based Metal-Organic framework as biodegradable nanozyme for microwave enhancing dynamic therapy, Biomaterials 214 (2019) 119223.

[28] X.G. Wang, Z.Y. Dong, H. Cheng, S.S. Wan, W.H. Chen, M.Z. Zou, J.W. Huo, H.X. Deng, X.Z. Zhang, A multifunctional metal-organic framework based tumor targeting drug delivery system for cancer therapy, Nanoscale 7(38) (2015) 16061-70.

[29] L. Liu, Y. Wei, S. Zhai, Q. Chen, D. Xing, Dihydroartemisinin and transferrin dual-dressed nanographene oxide for a pH-triggered chemotherapy, Biomaterials 62 (2015) 35-46.

[30] Z. Fan, H. Liu, Y. Xue, J. Lin, Y. Liao, Reversing cold tumors to hot: An immunoadjuvant-functionalized metal-organic framework for multimodal imaging-guided synergistic photo-immunotherapy, Bioactive Materials 6(2) (2021) 312-325.

[31] D. Wang, J. Zhou, R. Chen, R. Shi, G. Xia, S. Zhou, Z. Liu, N. Zhang, H. Wang, Z. Guo, Q. Chen, Magnetically guided delivery of DHA and Fe ions for enhanced cancer therapy based on $\mathrm{pH}$-responsive degradation of DHA-loaded Fe304@C@MIL-100(Fe) nanoparticles, Biomaterials 107 (2016) 88-101.

[32] Z. Zhang, X. Li, B. Liu, Q. Zhao, G. Chen, Hexagonal microspindle of NH2-MIL-101(Fe) metal-organic frameworks with visible-light-induced photocatalytic activity for the degradation of toluene, Rsc Adv 6(6) (2015) 4289-4295.

[33] X. Qian, J. Zhang, Z. Gu, Y. Chen, Nanocatalysts-augmented Fenton chemical reaction for nanocatalytic tumor therapy, Biomaterials 211 (2019) 1-13.

[34] J. Liu, Z. Luo, J. Zhang, T. Luo, J. Zhou, X. Zhao, K. Cai, Hollow mesoporous silica nanoparticles facilitated drug delivery via cascade $\mathrm{pH}$ stimuli in tumor microenvironment for tumor therapy, Biomaterials 83 (2016) 51-65.

[35] A. Hamdi, T.M. Roshan, T.M. Kahawita, A.B. Mason, A.D. Sheftel, P. Ponka, Erythroid cell mitochondria receive endosomal iron by a "kiss-and-run" mechanism, Biochim Biophys Acta 1863(12) (2016) 28592867.

[36] A. Terman, T. Kurz, Lysosomal iron, iron chelation, and cell death, Antioxid Redox Signal 18(8) (2013) 888-98.

[37] T. Liu, W. Liu, M. Zhang, W. Yu, F. Gao, C. Li, S.B. Wang, J. Feng, X.Z. Zhang, Ferrous-SupplyRegeneration Nanoengineering for Cancer-Cell-Specific Ferroptosis in Combination with Imaging-Guided Photodynamic Therapy, Acs Nano 12(12) (2018) 12181-12192.

[38] V. Trujillo-Alonso, E.C. Pratt, H. Zong, A. Lara-Martinez, C. Kaittanis, M.O. Rabie, V. Longo, M.W. Becker, G.J. Roboz, J. Grimm, M.L. Guzman, FDA-approved ferumoxytol displays anti-leukaemia efficacy against cells with low ferroportin levels, Nat Nanotechnol 14(6) (2019) 616-622. 
[39] T. Xu, Y. Ma, Q. Yuan, H. Hu, X. Hu, Z. Qian, J.K. Rolle, Y. Gu, S. Li, Enhanced Ferroptosis by OxygenBoosted Phototherapy Based on a 2-in-1 Nanoplatform of Ferrous Hemoglobin for Tumor Synergistic Therapy, Acs Nano 14(3) (2020) 3414-3425.

[40] T.A. Rouault, The role of iron regulatory proteins in mammalian iron homeostasis and disease, Nat Chem Biol 2(8) (2006) 406-14.

[41] J.L. Heath, J.M. Weiss, C.P. Lavau, D.S. Wechsler, Iron deprivation in cancer--potential therapeutic implications, Nutrients 5(8) (2013) 2836-59.

[42] S.V. Torti, F.M. Torti, Ironing out cancer, Cancer Res 71(5) (2011) 1511-4.

[43] Z.K. Pinnix, L.D. Miller, W. Wang, R. D'Agostino, Jr., T. Kute, M.C. Willingham, H. Hatcher, L. Tesfay, G. Sui, X. Di, S.V. Torti, F.M. Torti, Ferroportin and iron regulation in breast cancer progression and prognosis, Sci Transl Med 2(43) (2010) 43ra56.

[44] L.L. Dunn, Y. Suryo Rahmanto, D.R. Richardson, Iron uptake and metabolism in the new millennium, Trends Cell Biol 17(2) (2007) 93-100.

[45] A.T. McKie, D. Barrow, G.O. Latunde-Dada, A. Rolfs, G. Sager, E. Mudaly, M. Mudaly, C. Richardson, D. Barlow, A. Bomford, T.J. Peters, K.B. Raja, S. Shirali, M.A. Hediger, F. Farzaneh, R.J. Simpson, An ironregulated ferric reductase associated with the absorption of dietary iron, Science 291(5509) (2001) 17559.

[46] Hadi, Ranji-Burachaloo, Paul, Andrew, Gurr, Dave, Dunstan, Greg, Cancer Treatment Through Nanoparticle Facilitated Fenton Reaction, Acs Nano (2018).

[47] Z. Shen, T. Liu, Y. Li, J. Lau, Z. Yang, W. Fan, Z. Zhou, C. Shi, C. Ke, V.I. Bregadze, S.K. Mandal, Y. Liu, Z. $\mathrm{Li}, \mathrm{T}$. Xue, G. Zhu, J. Munasinghe, G. Niu, A. Wu, X. Chen, Fenton-Reaction-Acceleratable Magnetic Nanoparticles for Ferroptosis Therapy of Orthotopic Brain Tumors, Acs Nano 12(11) (2018) 11355-11365.

[48] T. Zhu, L. Shi, C. Yu, Y. Dong, F. Qiu, L. Shen, Q. Qian, G. Zhou, X. Zhu, Ferroptosis Promotes Photodynamic Therapy: Supramolecular Photosensitizer-Inducer Nanodrug for Enhanced Cancer Treatment, Theranostics 9(11) (2019) 3293-3307.

\section{Figures}


A

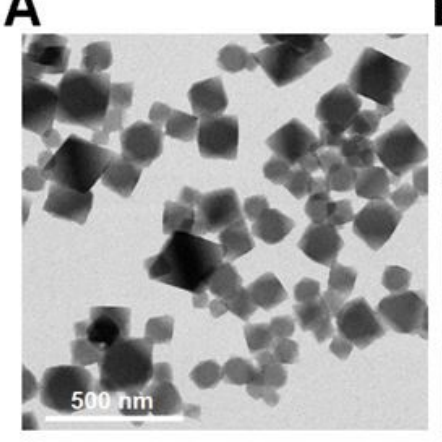

B

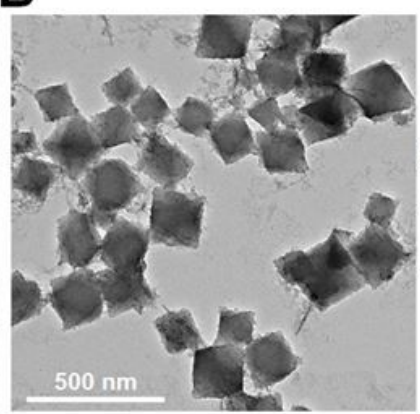

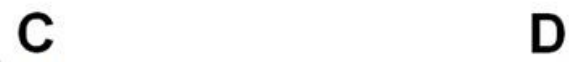

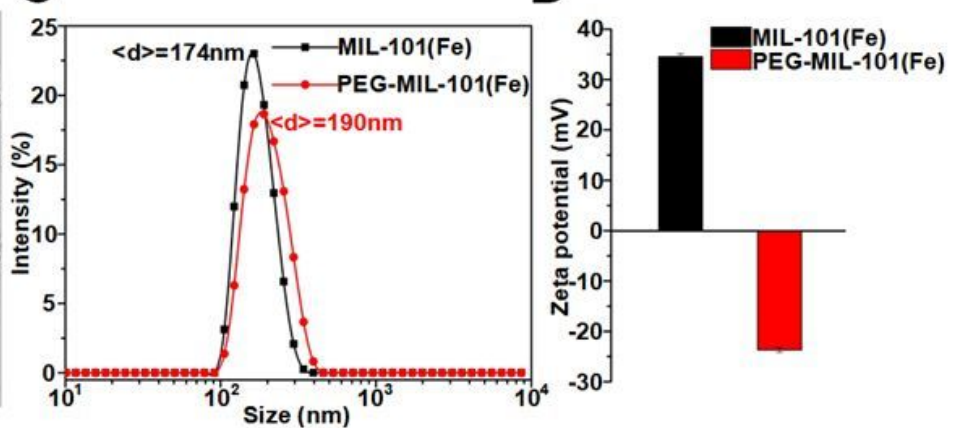

E
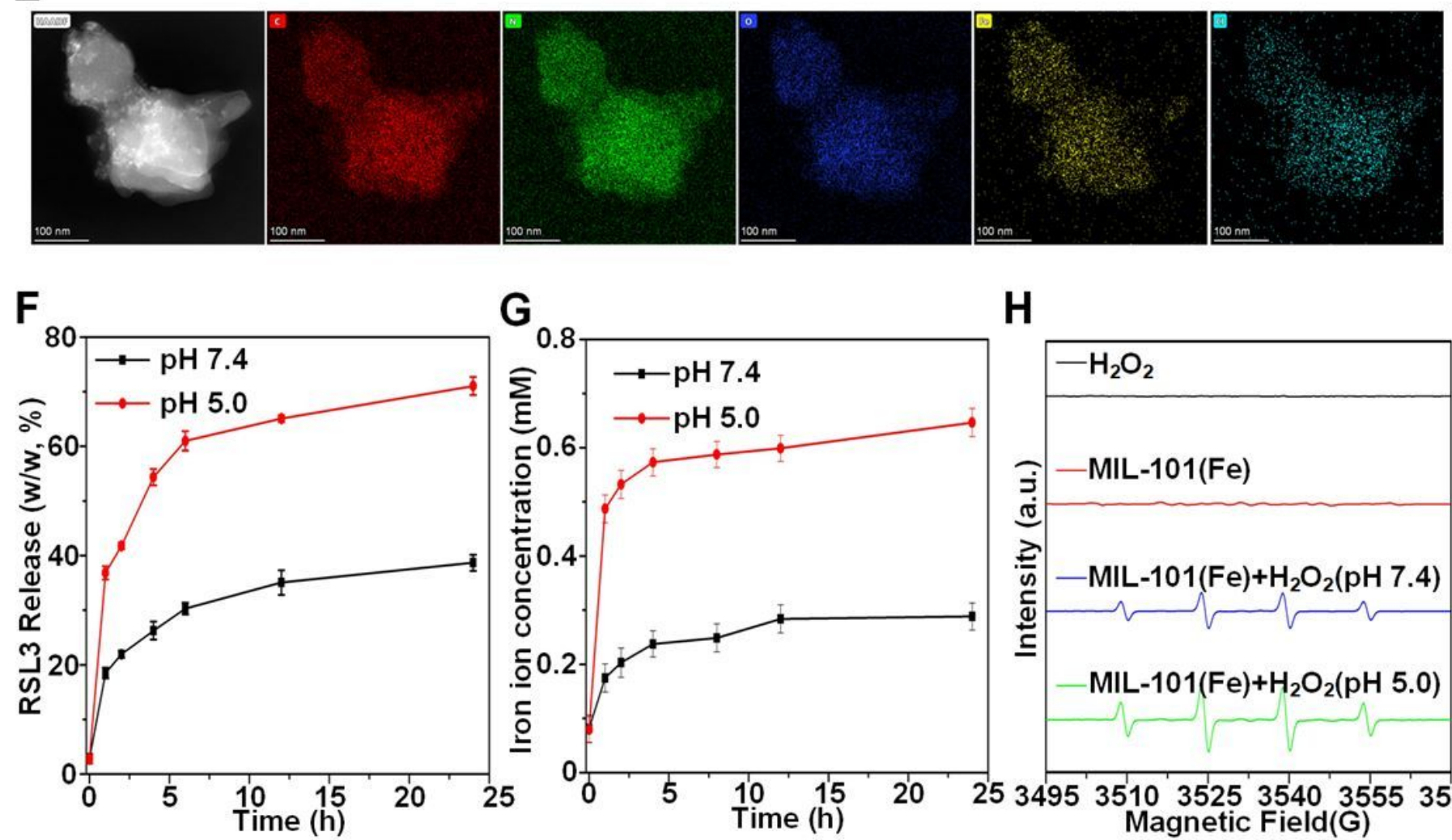

$\mathbf{G}$

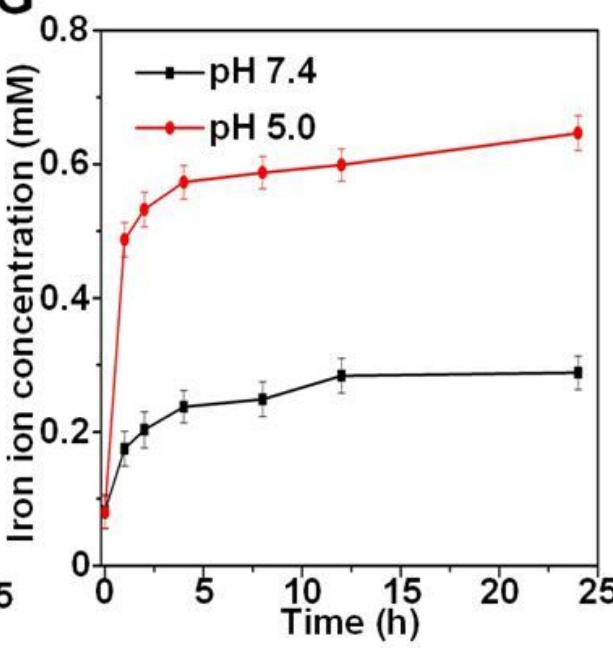

H

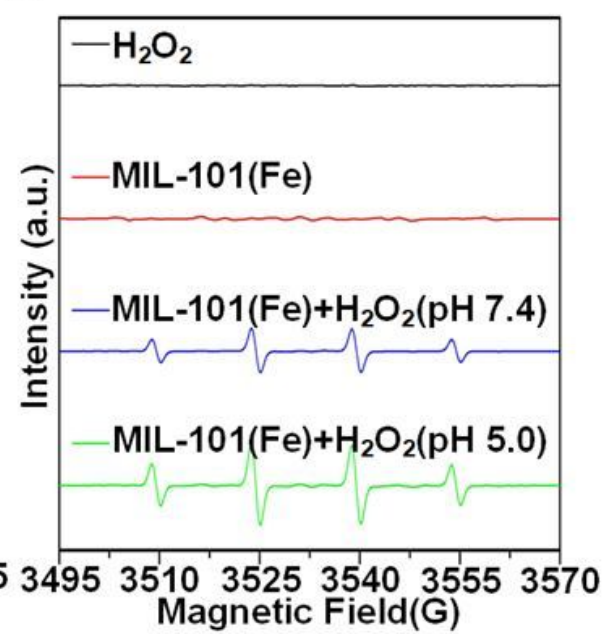

Figure 1

Characterization of MIL-101(Fe), PEG-MIL-101(Fe) and PEG-MIL-101(Fe)@RSL3. TEM images of (A) MIL101(Fe) NPs and (B) PEG-MIL-101(Fe) NPs. (C) DLS of MIL-101(Fe) and PEG-MIL-101(Fe) NPs. (D) Zeta potentials of MIL-101(Fe) and PEG-MIL-101(Fe) NPs. (E) Elemental mapping of C, Pt, O, Fe and Cl of PEGMIL-101(Fe)@RSL3 NPs. (F) Release of RSL3 and (G) iron from PEG-MIL-101(Fe)@RSL3 NPs under different $\mathrm{pH}$ conditions (7.4 and 5.0). (H) ESR spectra of different sample. 


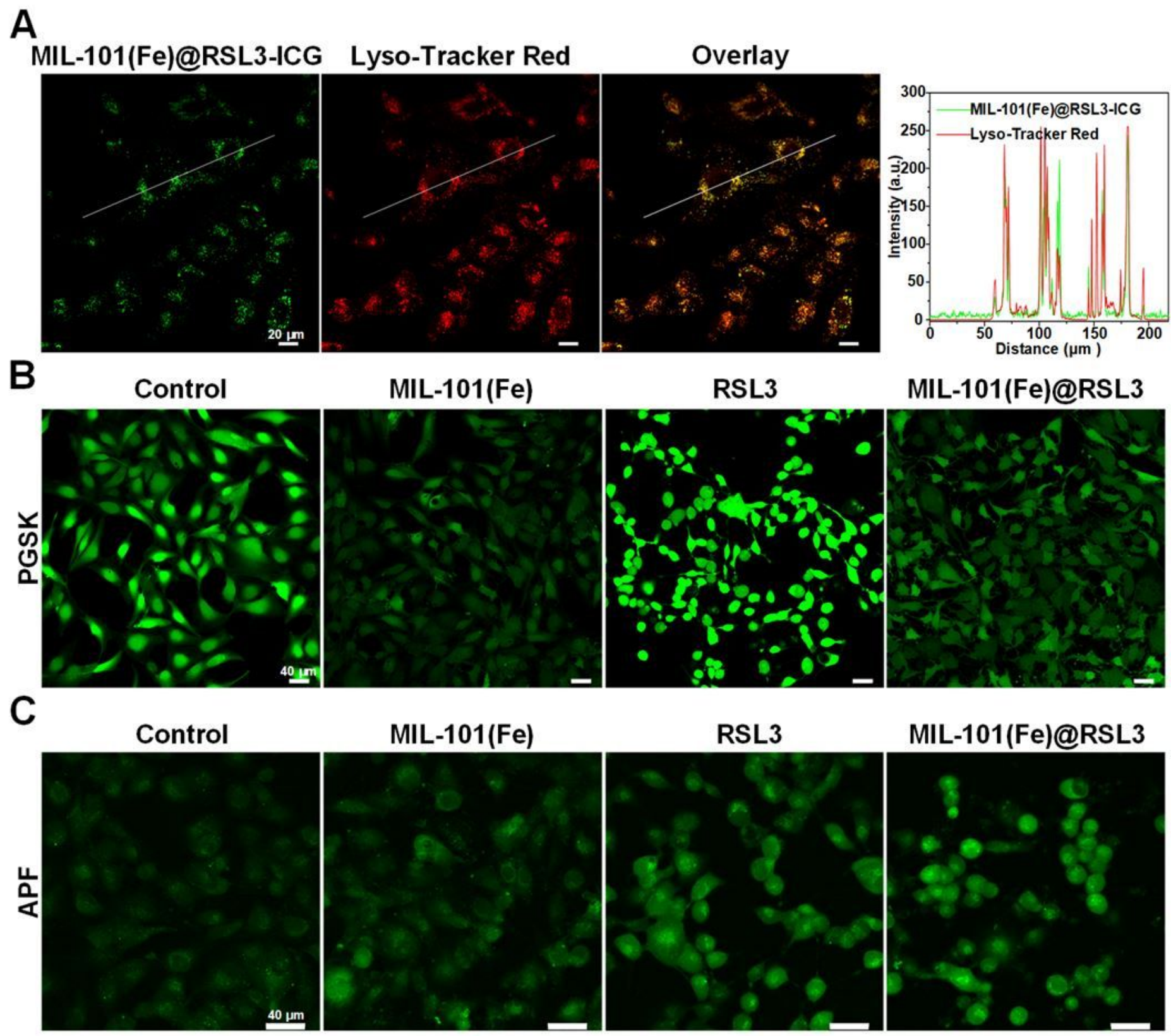

Figure 2

Intracellular iron ion release from MIL-101(Fe)@RSL3. (A) Intracellular localization of ICG-labelled MIL101(Fe)@RSL3 NPs in 786-0 cells. (B) Fluorescence images of PGSK-stained 786-0 cells with different treatments. (C) DCF fluorescence of cells. 


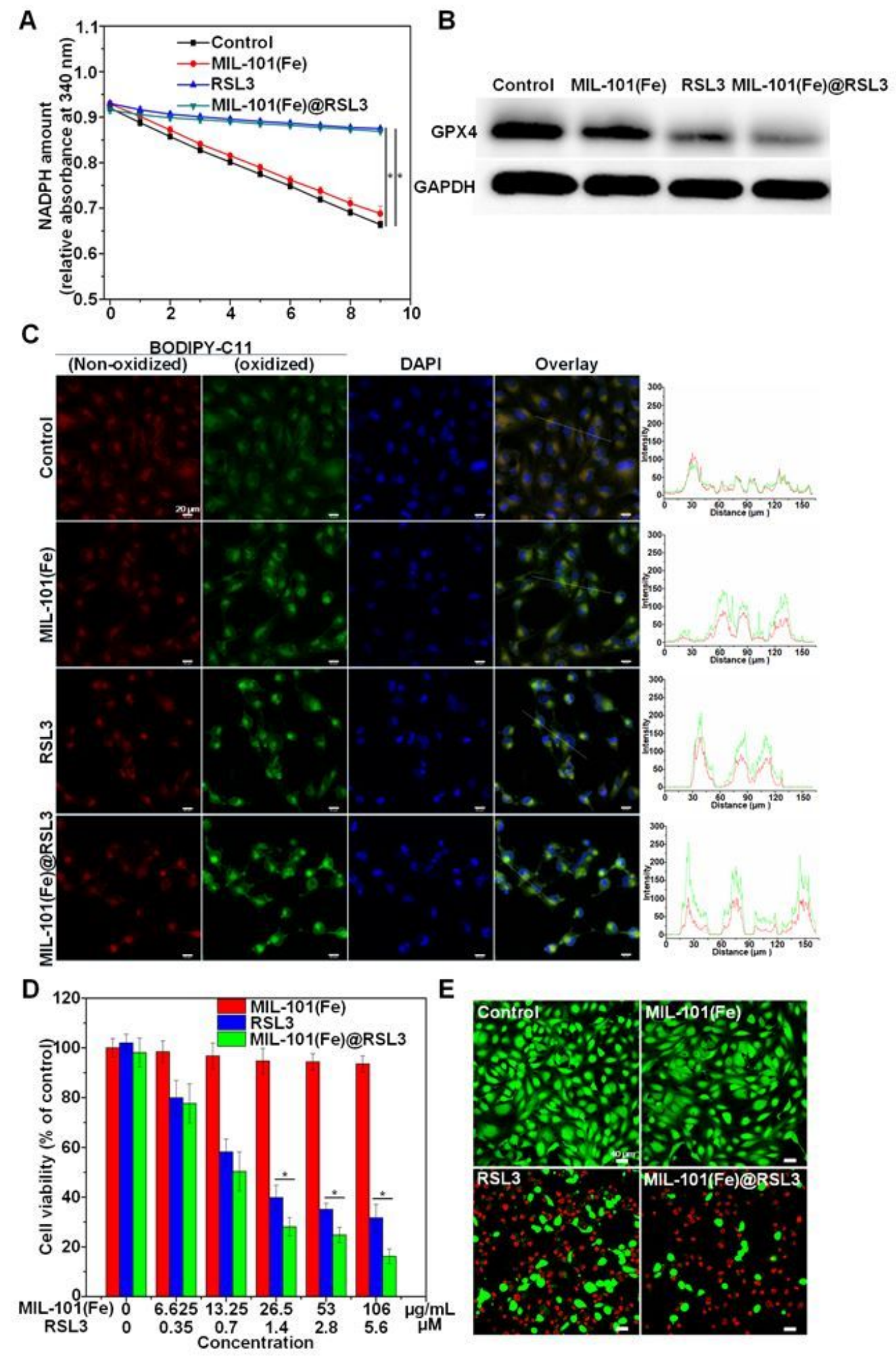

\section{Figure 3}

MIL-101(Fe)@RSL3 triggered ferroptosis in ccRCC cells. (A) GPX4 activity in 786-0 cells. The error bars represent the standard error of the mean of three replicates. P values: ${ }^{*} P<0.05$. (B) Western blots of GPX4 levels in 786-0 cells. (C) CLSM observations of cellular LPO with BODIPY581/591-C11 probe detection and the corresponding fluorescence intensity. (D) In vitro cytotoxicity of nanodrugs at different concentrations determined by CCK-8 proliferation assay. The error bars represent the standard error of the 
mean of six replicates. P values: ${ }^{*} P<0.05$. (E) Fluorescence images of $786-0$ cells co-stained with calceinAM (live cells, green) and PI (dead cells, red) after different treatments.
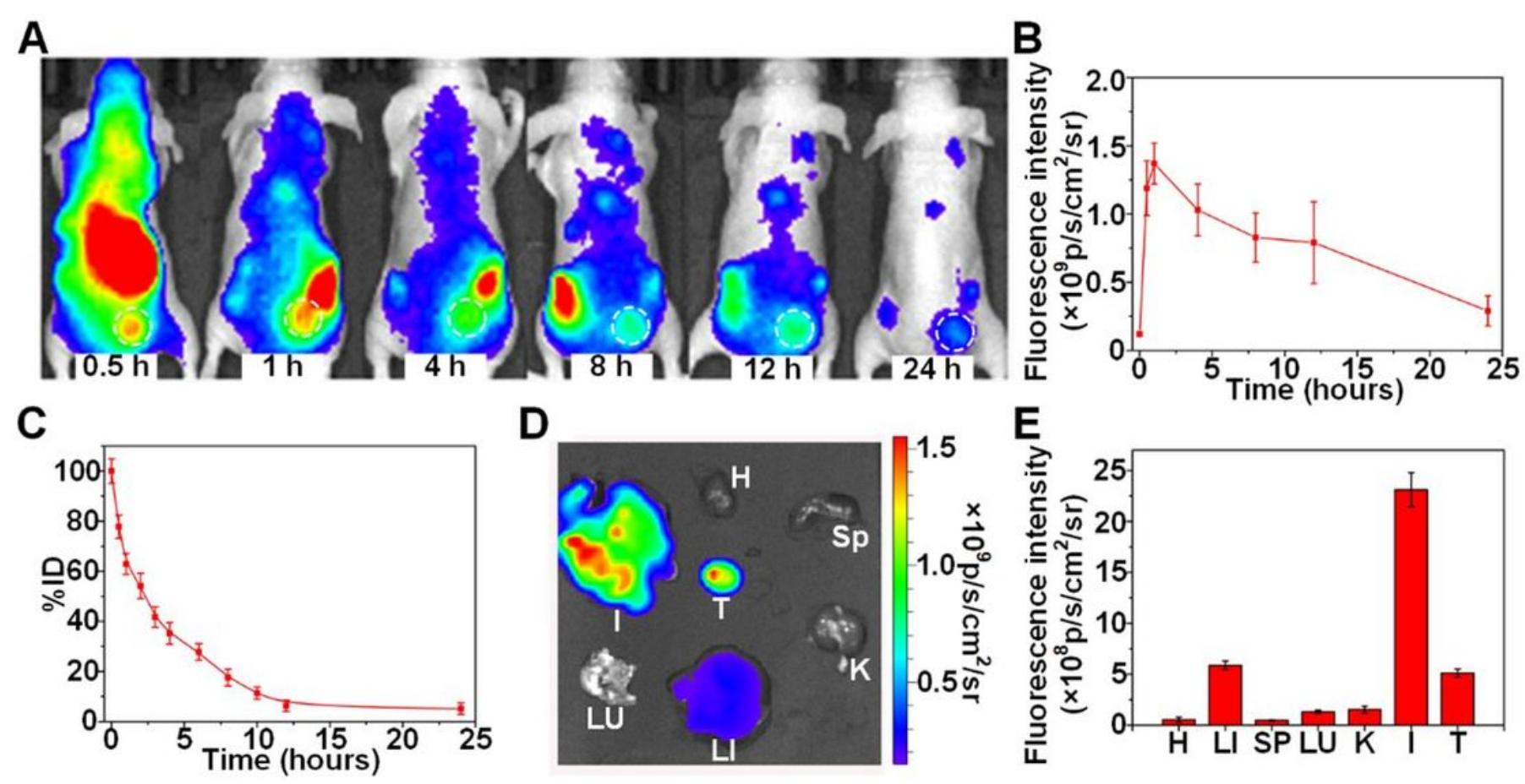

\section{Figure 4}

Biodistribution of ICG-MIL-101(Fe)@RSL3. (A) Fluorescence images of tumor-bearing mice at different time points post-injection of ICG-MIL-101(Fe)@RSL3. The circles indicate the tumor regions. (B) Corresponding fluorescence intensity of the tumor regions. (C) Blood circulation curve of ICG-MIL101(Fe)@RSL3 determined by measuring the ICG fluorescence in the blood at different time points postinjection ( $n=3)$. (D) The distribution of ICG-MIL-101(Fe)@RSL3 in the main organs and tumors of tumorbearing mice (I: intestine, $\mathrm{H}$ : heart, LU: lung, LI: liver, K: kidney, SP: spleen, and T: tumor) and (E) the corresponding fluorescence intensity of the organs $(n=3)$. 

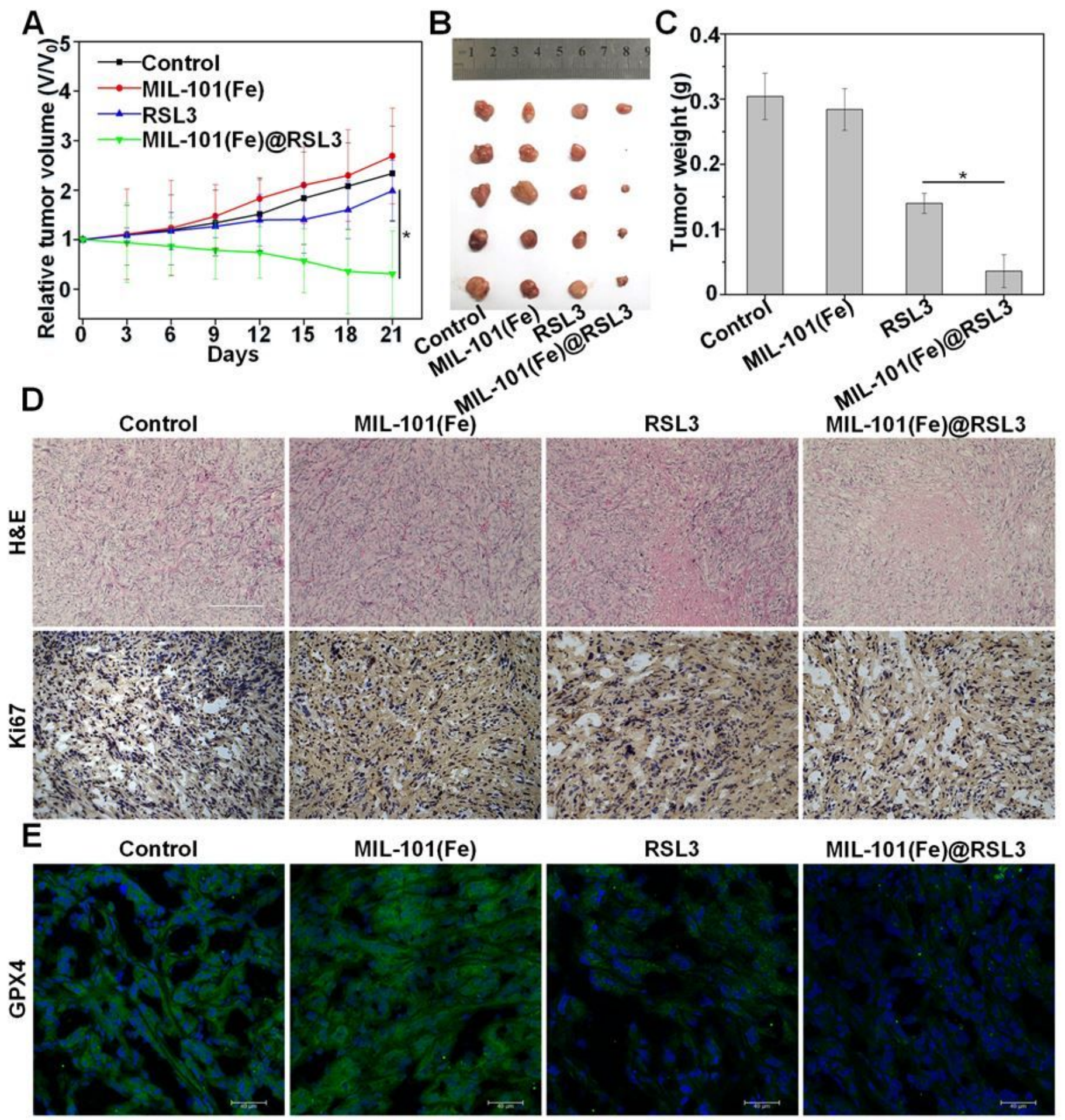

Figure 5

In vivo therapeutic efficacy of MIL-101(Fe)@RSL3 NPs. (A) Representative photographic images of mice with different treatments. (B) Relative tumor volumes after different treatments $(n=6)$. (C) Photographic images of tumors and (D) corresponding tumor weights in different treatment groups at 21 days posttreatment $(n=6)$. (E) H\&E stained images of tumor tissue slices from different treatment groups. ${ }^{*}<<0.05$. 

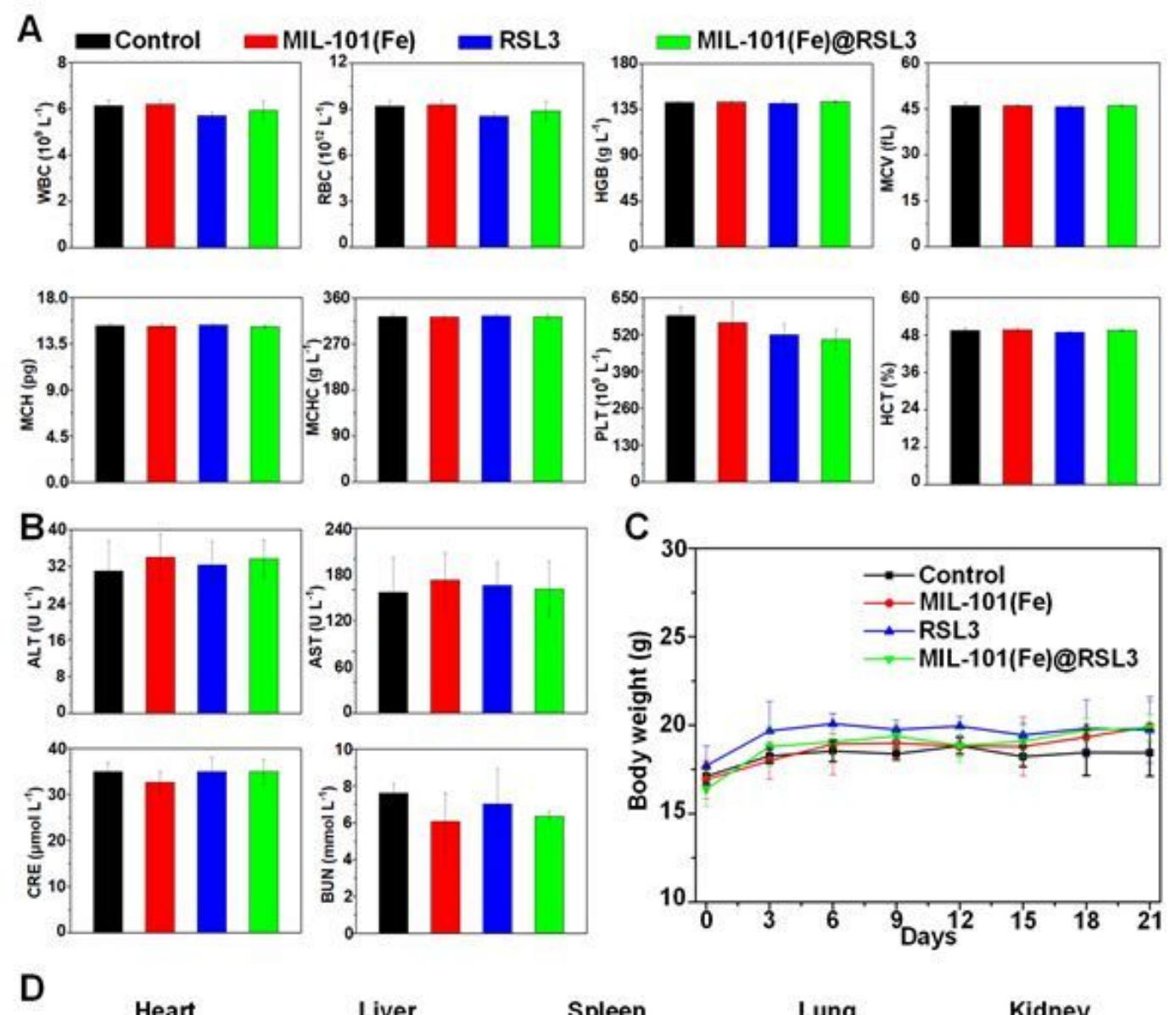

Liver Spleen Lung Kidney

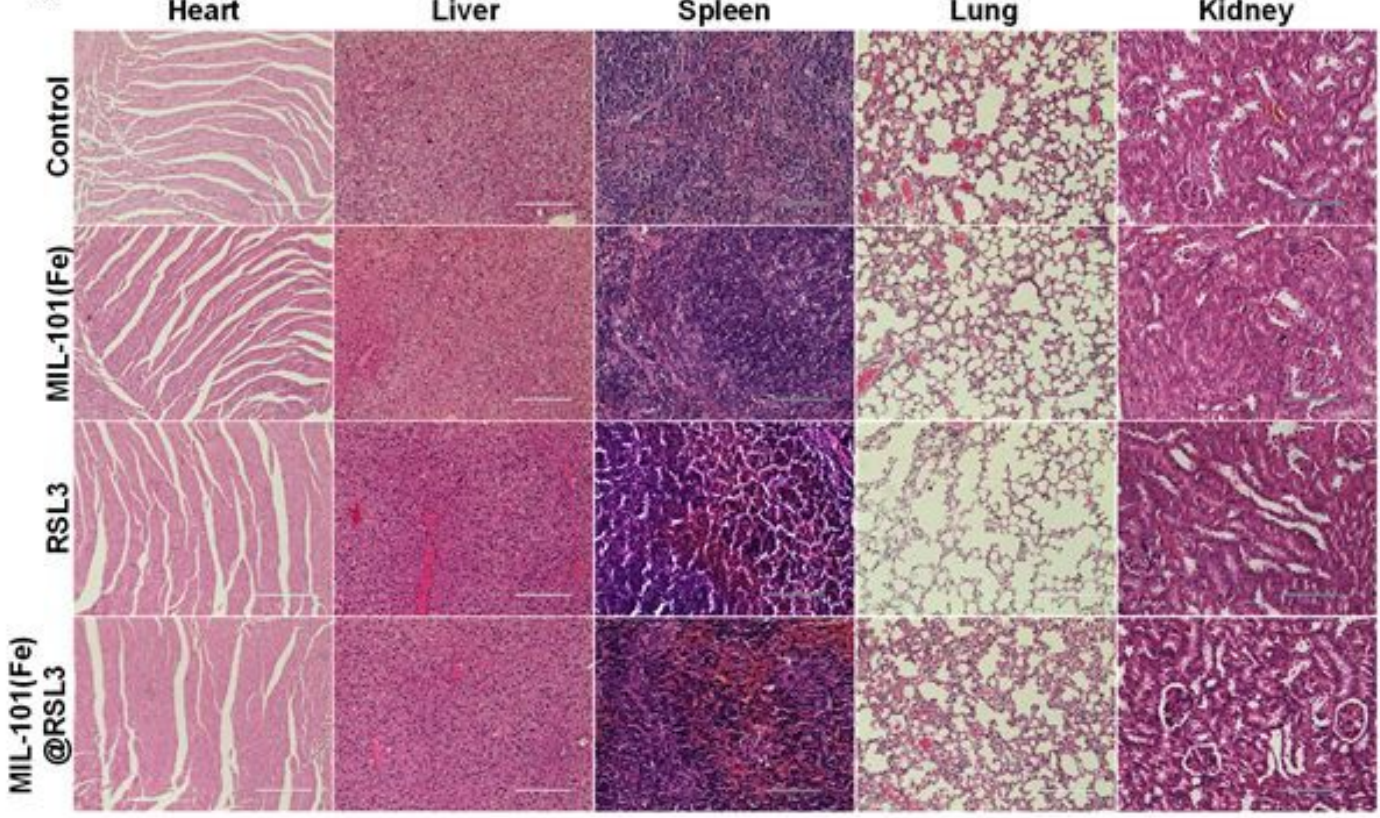

\section{Figure 6}

Side effects evaluation. (A) Haematological data of the mice after different treatments. The terms are as follows: white blood cell (WBC), red blood cell (RBC), haemoglobin (HGB), mean corpuscular volume $(\mathrm{MCV})$, mean corpuscular haemoglobin $(\mathrm{MCH})$, mean corpuscular haemoglobin concentration (MCHC), platelet (PLT) and haematocrit (HCT). (B) Blood biochemical analysis. (C) The body weights of the mice were measured during the 21-day evaluation period under different conditions. (D) Representative H\&E- 
stained sections of the major organs (heart, liver, spleen, lung, and kidney). Data presented as the mean \pm $\operatorname{SD}(n=6)$.

\section{Supplementary Files}

This is a list of supplementary files associated with this preprint. Click to download.

- scheme1.jpg

- si.docx 